\title{
Uterovaginal anastomosis for the management of congenital atresia of the uterine cervix
}

\author{
Anish Keepanasseril • S. C. Saha $\cdot$ Rashmi Bagga • \\ Sameer Vyas $\cdot$ L. K. Dhaliwal
}

Received: 15 September 2010 /Accepted: 3 November 2010/Published online: 16 December 2010

(C) Springer-Verlag 2010

\begin{abstract}
Congenital atresia of the cervix is a rare mullerian anomaly. Hysterectomy has been advocated as the management of choice in the early days as the reproductive performance is thought to be low despite successful neo-canal creation. In recent years, conservative surgery is being recommended more frequently in patients with congenital cervical atresia and with total or partial vaginal aplasia and is shown to have a better reproductive performance. The treatment strategy should be tailored to relieve retrograde menstrual symptoms and restore fertility. Here, we report a young girl with congenital cervical atresia with upper vaginal atresia managed with uterovaginal anastomosis and review the management options and reproductive performance in such cases.
\end{abstract}

Keywords Cervical Atresia - Uterovaginal anastomosis . Mullerian Anomaly

\section{Background}

Cervical atresia is an extremely rare and complex mullerian malformation. Unlike most other mullerian anomalies, the initial surgical management of congenital cervical atresia

A. Keepanasseril $\cdot$ S. C. Saha $(\bowtie) \cdot$ R. Bagga $\cdot$ L. K. Dhaliwal Department of Obstetrics and Gynecology, Postgraduate Institute of Medical Education and Research (PGIMER),

Sector-12,

Chandigarh, India 160012

e-mail: drscsaha@gmail.com

\section{S. Vyas}

Department of Radiodiagnosis, Postgraduate Institute of Medical Education and Research (PGIMER),

Sector-12,

Chandigarh, India 160012 remains controversial. Total hysterectomy eliminates the symptoms related to hematometra, but loss of reproductive function is irreversible. Hysterectomy should be avoided as first choice and reserved for cases where canalization attempts fail or are impossible. However, canalization procedures, mainly cervical drilling, may be followed by recurrent obstruction of the uterovaginal neo-canal, and persistent infertility. Hence, a majority of clinicians view hysterectomy as the optimal primary surgical management in these patients $[1,2]$. Recently, small series have been reported which show an improved reproductive performance after utero-vaginal anastomosis [3, 4]. We report a 16-yearold girl who underwent uterovaginal anastomosis for cervical atresia and review the management options as well as the reproductive performance in such patients.

\section{Method}

A 16-year-old girl with primary amenorrhea and cyclic pelvic pain attended our hospital in January 2009. Her clinical examination results showed appropriate general feminization, normal external genitalia; rectal examination results revealed a small uterus with unremarkable adnexa. Trans-abdominal sonography evidenced the presence of uterus $(4.2 \times 2.9 \times 3 \mathrm{~cm})$ with suspicion of an absent cervix with normal ovaries and kidneys. Magnetic resonance imaging (MRI) revealed that the uterus and endometrial cavity were normal with cervix and the upper end of the vagina suspected to be aplastic (Figs. 1 and 2). The lower vagina was normal and a diagnosis of cervical and upper vaginal atresia was made. Her examination under anesthesia revealed the length of patent lower vagina to be about $6 \mathrm{~cm}$. She underwent uterovaginal anastomosis by an abdominoperineal approach; adhering to the microsurgical principles. 
Fig. 1 Sagittal T2-weighted images $(\mathbf{a}, \mathbf{b})$ of pelvis showing hypoplasia/atresia of cervix and upper vagina (black arrow) Uterine body is normal in contour and shows normal zonal demarcation (white arrow)

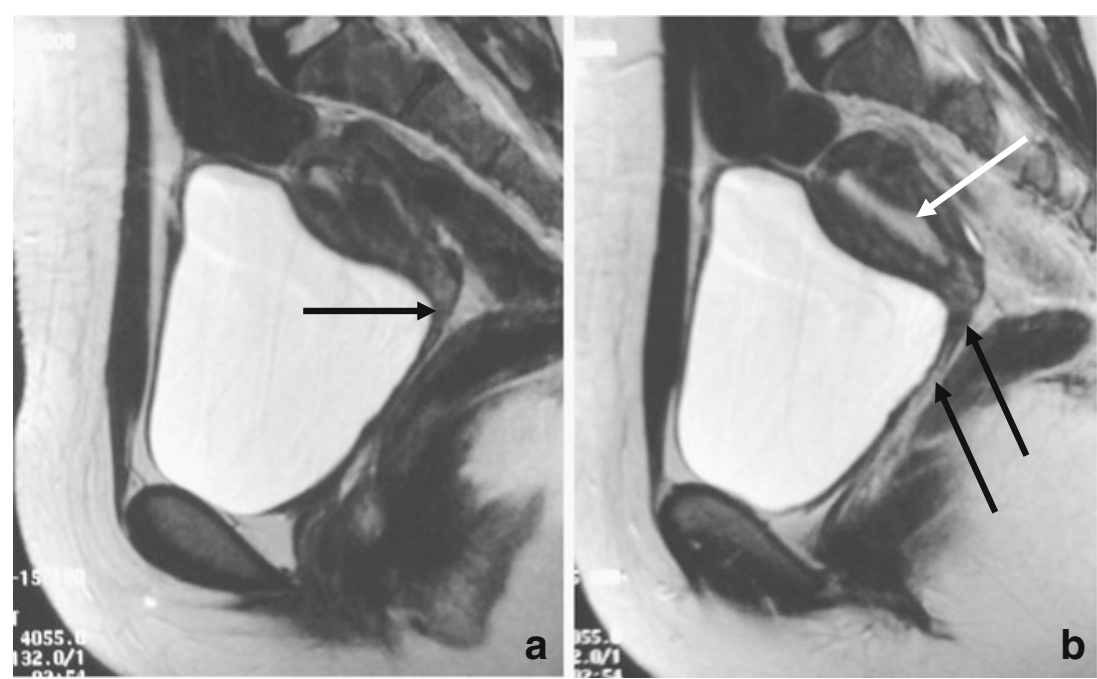

The uterovesical fold of peritomeum was incised and the bladder was reflected down over the atretic cervix (which was about $1.5 \mathrm{~cm}$ long) and the upper vagina. A $1-\mathrm{cm}$ transverse incision was made at the uppermost portion of the patent vagina. Next, a $1-\mathrm{cm}$ vertical incision was made in the lower end of the uterine body just above the atretic cervix and the endometrial cavity was opened. A hegar dilator (number 7-8) was introduced into the endometrial cavity through the uterine incision which was extended over the dilator to reach upto the superior limit of the atretic cervix. The dilator was removed and an 18-Fr Foleys catheter was introduced into the vagina from below, made to exit through the vaginal incision and enter into the endometrial cavity through the uterine incision, anterior to and bypassing the atretic cervix. With the Foleys catheter in place, the edges of the uterine incision were sutured to the edges of the vaginal incision. Thus, the lower portion of the uterus was anastomosed to the uppermost part of the patent vagina, bypassing the atretic cervix which was retained as the posterior lip of the neo-cervix, to the neo-uterovaginal canal.
A small endometriotic cyst $(2 \times 2 \mathrm{~cm})$ in the left ovary was managed with cystectomy. The post-operative period was normal. Antibiotic treatment was given for 2 weeks in view of the retained Foleys catheter. She was given cyclical low dose contraceptive pills for 6 months. She had normal regular period in the month following surgery. The Foleys catheter was expelled spontaneously after the first menstrual cycle (3 weeks after surgery). Presently, 9 months after surgery, she is having spontaneous and regular menstrual cycles without any dysmenorrhea.

\section{Findings}

Atresia or agenesis of the uterine cervix is a rare developmental malformation of the female genital tract. Fujimoto et al. [5] reported 51 cases in the world literature. According to the Buttram and Gibbons [6] classification, cervical agenesis represents a Class I B mullerian anomaly

Fig. 2 Axial T2-weighted images $(\mathbf{a}, \mathbf{b})$ of pelvis showing uterine body with normal zonal demarcation and endometrial cavity (a). Cervix is hypoplastic/ atretic (black arrow)
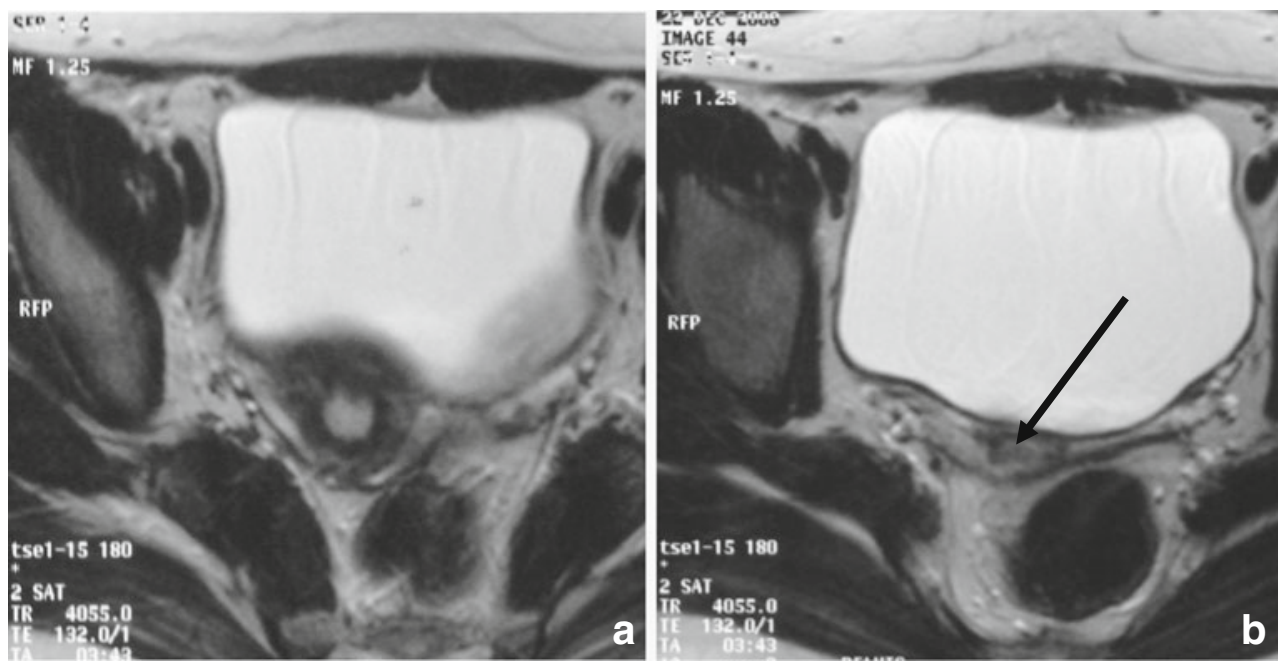
whereas the ASRM classification of 1988 places it in Class II b. In approximately $50 \%$ of cases, cervical agenesis is associated with partial or complete vaginal agenesis [5].

Patients usually present with amenorrhea and symptoms of retrograde menstruation plus hematometra. In pubescent girls, pelvic pain in the absence of menstrual bleeding must evoke the suspicion of an obstructive genital syndrome. Clinical examination eliminates vaginal atresia or an imperforate hymen, but it may not always be possible to differentiate cervical atresia from a high vaginal septum. Transabdominal or transperineal sonography may help to identify the level of obstruction but is not so reliable in the diagnosis of cervical atresia [7,8]. The MRI is a more reliable imaging technique, and may diagnose associated upper genital tract anomalies or problems like hematosalpinx and endometriosis [9, 10]. Laparoscopy allows an exploration of the pelvis with assessment of the internal genital organs. An intravenous pyelography (IVP) can be performed because of the frequency of urinary tract malformations associated with Mullerian ducts anomalies [4]. MRI may be sufficient to obtain a preoperative diagnosis of cervical atresia, as in the present patient. MRI may also diagnose urinary tract malformations; hence an IVP may be omitted.

Unlike most of the other mullerian anomalies, management of congenital cervical atresia is challenging. The earliest reported case of congenital cervical atresia managed by hysterotomy and cervical canalization was described in 1900 [5]. The most successful surgical methods employed involved a transvaginal or transabdominal approach to create a neo-ostium through the dense fibrous cervix and communicate it to the endometrial cavity and vagina. Patency was maintained by application of stents (which were retained postoperatively for varying periods of time), with or without a surrounding full or split-thickness skin graft. However, re-stenosis of fibrous tissue, postoperative severe infection, or septicemia in occasional cases resulted in the recommendation of hysterectomy for this problem [4, 11-13]. However, in recent years, there has been resurgence towards conservative surgery which is now being frequently attempted in congenital cervical atresia associated with total or partial vaginal aplasia, with an aim to preserve the reproductive capability and relieve the menstrual symptoms. A procedure that sustains cyclic menses and does not allow re-stenosis would be optimal. Canalization techniques such drilling are easier to perform, but these are associated with re-stenosis of the cervix and its complications in nearly $40-60 \%$. Hence, the technique of utero-vaginal anastomosis described by Deffarges et al. [4] is now preferred as it has a lower risk of re-stenosis and can be performed even in the presence of associated vaginal aplasia. It reduces the risk of damage to the bladder and rectum and obtains a reliable canalization of uterovaginal canal. They reported only one patient out of 18 who developed re-stenosis of the uterovaginal canal leading to multiple canalization procedures, and ultimately needed salpingo-oophorectomy for pyosalpinx. Susbequently techniques using laproscopy or with laproscopic assistance have been reported. Novel techniques have been described by El Saman using laproscopic assistance (i.e., endoscopic monitored canaisation under vaginoscopic monitoring and retropubic balloon vagnioplasty). It has the advantages of requiring less laproscopic expertise and with less perineal dissection or extensive $[14,15]$. However, the long-term outcome and the reproductive performances after the laparoscopic techniques are yet to be reported.

Due to the risk of retrograde menstruation and subsequent development of endometriosis, creating an outflow tract should be done as early as possible (12-16 years). In cases where hysterectomy needs to be performed for any associated pathology, creation of the neovagina may be delayed up to the age of 18-20 years. In complete aplasia of the vagina, such a two-stage procedure has been carried out successfully [16, 17].

The success of uterovaginal anastomosis depends upon the size of the created ostium, the length of the new endocervical canal, the presence of vaginal mucosa adjacent to the end of the neo-ostium, and the duration of stenting [18]. Laparoscopically assisted utero-vestibular anastomosis is also reported, but data about post procedure reproductive performance is yet not available [19]. The duration of the stenting has been reported to range from 3 weeks to 3 months, but in the series reported by Deffarges et al. [4], it was kept for 2 weeks only, and re-stenosis occurred in only $1 / 18$. In our case, we chose uterovaginal anastomosis, as the long-term outcome and the reproductive performance after this method were reported in the literature. She is currently relieved of her menstrual symptoms. Her stent (Foleys catheter) was expelled spontaneously after 3 weeks, and she is symptom-free 9 months post-procedure.

The likelihood of spontaneous pregnancy occurring as a result of canalization appears to be low. This low fertility rate may be due to the presence of associated endometriosis and pelvis adhesions, plus the lack of normal endocervical canal glandular function. Very few spontaneous pregnancies have been reported after canalization of a completely or partially atretic cervix [4]. Assisted reproductive techniques are usually required to help these patients to achieve a pregnancy. [20, 21]. The need of cervical cerclage is described but is controversial. In series by Deffarges et al. [4], $40 \%$ of the women who attempted to conceive had a successful pregnancy, and only one out o ten patients had cerclage. They should be delivered by elective cesarean as the vertical uterine incision may increase the chance of uterine rupture. 


\section{Conclusion}

In conclusion, a conservative approach may be recommended for women with congenital cervical atresia even in the presence of vaginal agenesis. Uterovaginal anastomosis can be considered as the surgical method of choice in these women. Surgery should be performed as early as possible to avoid complications like endometriosis and its sequelae which may hamper future reproductive performance. In addition, the presence of regular menses has an extremely favorable psychological impact on these young women.

Conflict of interest The authors report no conflicts of interest. The authors alone are responsible for the content and writing of the paper.

\section{References}

1. Rock JA, Schlaff WD, Zacur HA, Jones HW Jr (1984) The clinical management of congenital absence of the uterine cervix. Int J Gynaecol Obstet 22:231-235

2. Fliegner JR, Pepperell RJ (1994) Management of vaginal agenesis with a functioning uterus. Is hysterectomy advisable? Aust $\mathrm{N} \mathrm{Z} \mathrm{J}$ Obstet Gynaecol 34:467-470

3. Singh J, Devi YL (1983) Pregnancy following surgical correction of nonfused müllerian bulbs and absent vagina. Obstet Gynecol 61:267-269

4. Deffarges JV, Hadda B, Musset R, Paniel BJ (2001) Utero-vaginal anastomosis in women with uterine cervix atresia: long-term follow-up and reproductive performance. A study of 18 cases. Hum Reprod 16:1722-1725

5. Fujimoto VY, Miller JH, Klein NA, Soules MR (1997) Congenital cervical atresia: report of seven cases and review of the literature. Am J Obstet Gynecol 177:1419-1425

6. Buttram VJ, Gibbons W (1979) Mullerian anomalies: a proposed classification (an analysis of 144 cases). Fertil Steril 32:40-46

7. Graham D, Nelson MW (1986) Combined perineal-abdominal sonography in the evaluation of vaginal atresia. J Clin Ultrasound $14: 735-738$
8. Meyer WR, McCoy MC, Friz MA (1995) Combined perinealabdominal sonography in the evaluation of transverse vaginal septum. Obstet Gynecol 85:882-884

9. Reinhold C, Hricak H, Forstner R, Ascher SM, Bret PM, Meyer WR, Semelka RC (1997) Primary amenorrhea: evaluation with MR imaging. Radiology 203:383-390

10. Lang IM, Babyn P, Oliver GD (1999) MR imaging of pediatric uterovaginal anomalies. Pediatr Radiol 29:163-170

11. Olive DL, Henderson DY (1987) Endometriosis and mullerian anomalies. Obstet Gynecol 69:412-415

12. Jacob JH, Griffin WT (1989) Surgical reconstruction of the congenitally atretic cervix: two cases. Obstet Gynecol Surv 44:556-569

13. Hovsepian DM, Auyeung A, Ratts VS (1999) A combined surgical and radiologic technique for creating a functional neoendocervical canal in a case of partial congenital cervical atresia. Fertil Steril 71:158-162

14. El Saman AM (2009) Combined retropubic balloon vaginoplasty and laparoscopic canalization. Am J Obstet Gynecol 201:333.e1333.e5

15. El Saman (2010) Endoscopically monitored canalization for treatment ofcongenital cervical atresia: the least invasive approach. Fertil Steril 94:313-316

16. Bugmann P, Amaudruz M, Hanquinet S, La Scala G, Birraux J, Le Coultre C (2002) Uterocervicoplasty with a bladder mucosa layer for the treatment of complete cervical agenesis. Fertil Steril 77:831-835

17. Acién P, Acién MI, Quereda F, Santoyo T (2008) Cervicovaginal agenesis: spontaneous gestation at term after previous reimplantation of the uterine corpus in a neovagina: Case Report. Hum Reprod 23:548-553

18. Gurbuz A, Karateke A, Haliloglu B (2005) Abdominal surgical approach to a case of complete cervical and partial vaginal agenesis. Fertil Steril 84:217

19. Fedele L, Bianchi S, Frontino G, Berlanda N, Montefusco S, Borruto F (2008) Laparoscopically assisted uterovestibular anastomosis in patients with uterine cervix atresia and vaginal aplasia. Fertil Steril 89:212-216

20. Nargund G, Parsons J (1996) A successful in-vitro fertilization and embryo transfer treatment in a woman with previous vaginoplasty for congenital absence of vagina. Hum Reprod 11:1654

21. Anttila L, Penttilä TA, Suikkari AM (1999) Successful pregnancy after in-vitro fertilization and transmyometrial embryo transfer in a patient with congenital atresia of cervix: case report. Hum Reprod 14:1647-1649 